\title{
Nonlinear repulsive force between two solids with axial symmetry
}

\author{
Diankang Sun, ${ }^{1}$ Chiara Daraio, ${ }^{2}$ and Surajit $\operatorname{Sen}^{1}$ \\ ${ }^{1}$ Department of Physics, State University of New York, Buffalo, New York 14260-1500, USA \\ ${ }^{2}$ Departments of Applied Physics and Aeronautics, MS 105-50, California Institute of Technology, Pasadena, California 91125, USA
}

(Received 21 November 2010; revised manuscript received 13 March 2011; published 20 June 2011)

\begin{abstract}
We modify the formulation of Hertz contact theory between two elastic half-solids with axial symmetry and show that these modifications to Hertz's original framework allow the development of force laws of the form $\mathcal{F} \propto z^{n}, 1<n<\infty$, where $\mathcal{F}$ is the force and $z$ is the distance between the centers of the two solids. The study suggests that it may be possible to design physical systems that can realize such force laws. We let the half-solids be characterized by radii of curvatures $R_{1}$ and $R_{2}$ and invoke a factor $m>0$ to describe any aspect ratio in the two bodies, all being valid near the contact surface. We let the $x-y$ plane be the contact surface with an averaged pressure across the same as opposed to a pressure profile that depends on the contact area of a nonconformal contact as originally used by Hertz. We let the $z$ axis connect the centers of the masses and define $z_{1,2}=x^{\alpha} / R_{1,2}^{\alpha-1}+y^{\alpha} /\left(m R_{1,2}\right)^{\alpha-1}$, where $z_{1,2} \geqslant 0$ refers to the compression of bodies $1,2, \alpha>1, m>0$, $x, y \geqslant 0$. The full cross section can be generated by appropriate reflections using the first quadrant part of the area. We show that the nonlinear repulsive force is $F=a z^{n}$, where $n \equiv 1+1 / \alpha$, and $z \equiv z_{1}+z_{2}$ is the overlap and we present an expression for $a=f\left(E, \sigma, m, \alpha, R_{1}, R_{2}\right)$ with $E$ and $\sigma$ as Young's modulus and the Poisson ratio, respectively. For $\alpha=2, \infty$, to similar geometry-dependent constants, we recover Hertz's law and the linear law, describing the repulsion between compressed spheres and disks, respectively. The work provides a connection between the contact geometry and the nonlinear repulsive law via $\alpha$ and $m$.
\end{abstract}

DOI: 10.1103/PhysRevE.83.066605

PACS number(s): 46.40.Cd, 43.25.+y, 45.70.-n

\section{INTRODUCTION}

The repulsive force between two elastic, paraboloidal solids with axial symmetry in intimate contact is known to be strongly nonlinear [1]. The topic has a rich history dating back to Hertz [1] and others (e.g., see [2-6]). The existence of the Hertz force has important consequences and here are two examples. A gently perturbed $1 D$ chain of spherical grains is known to admit a new kind of solitary wave propagation [7,8]. The propagation of mechanical energy at shallow depths in dry granular beds depend on the force chains that span the granular fabric [9] and remains an area of broad interest in many contexts such as landmine detection [10], shock absorption [11], and thermal conductivity especially in the context of geothermal applications [12] and geophysics at ultrashallow depths [13].

Crucial to the existing studies on the derivation of the Hertz force for nonconformal contacts are two points: (i) the contact interface between the two solids is, in general, elliptical in nature, e.g., with major $(x)$ and minor $(y)$ axis lengths of $a$ and $b$ and (ii) the pressure in the contact area decays as $\left[1-(x / a)^{2}-(y / b)^{2}\right]^{1 / 2}$ from the highest value at the center to zero at the edge of the ellipse. A necessary step is also that the cross section of the contact region is significantly smaller than the cross-sectional area of the elastic bodies at their centers (see, for example, in [6]). The stress-strain relation via Young's modulus is next used to establish Hertz's law.

There is a large body of literature on the precise form of Hertz's law as well as on different pressure profiles that may be relevant for actual applications [14]. In addition, there is a formidable interest in tribology on cases of conformal contact, i.e., for contacts with multiple asperities [15]. While all the treatments agree on the force-displacement behavior, the prefactors obtained depend on (i) and (ii) above. The simplest form of Hertz's law for two elastic bodies with paraboloidal surfaces in the vicinity of the contact point is explicitly derived in Ref. [4]. The distance $L$ separating the undeformed surfaces is characterized by $L=A x^{2}+B y^{2}$ where $A, B$ depend on the radii of curvature of the paraboloids. For an elliptical contact if $a$ and $b$ are the semimajor and semiminor axes, respectively, we let $\kappa \equiv a / b$. Then the Hertz force has been shown to be $\mathcal{F}(z)=k z^{3 / 2}$, where $k=$ $\frac{4}{3} \frac{q_{a}^{3 / 2}}{\left(D_{1}+D_{2}\right) \sqrt{A+B}} /\left[\int_{0}^{\infty} \frac{d \xi}{\sqrt{(1+\xi)\left(\kappa^{-2}+\xi\right) \xi}}\right]^{3 / 2}$, where $z$ represents the overlap function between the two spheres, $D_{1,2}=\frac{1-\sigma_{1,2}^{2}}{E_{1,2}}$, and $q_{a}=\left(\phi_{1}+\phi_{2}\right)^{1 / 3}, \phi_{1}=\int_{0}^{\infty} \frac{d \xi}{\sqrt{(1+\xi)^{3}\left(\kappa^{-2}+\xi\right) \xi}}$ and $\phi_{2}=$ $\int_{0}^{\infty} \frac{d \xi}{\sqrt{(1+\xi)\left(\kappa^{-2}+\xi\right)^{3} \xi}}[1,4]$. The general properties of the prefactor are described in pp. 195-198 in Ref. [2] and in some detail in pp. 86-87 in Ref. [4]. The overlap function $z$ is defined as the distance between the centers of the spheres when they are barely in contact, i.e., $\left(R_{1}+R_{2}\right)$ less the same distance when they are pressed against one another (say, $d$ ) and hence the variable $z \equiv R_{1}+R_{2}-d \geqslant 0$. It is important to note here that explicit knowledge of $\kappa$ is needed from experiments to determine $k$.

Though much remains to be learned about systems with nonlinear forces, Fermi et al. showed that one dimensional systems with nonlinear forces may have unique energy transport characteristics [16]. Studies have considered energy transport in a variety of simple nonlinear systems (see, e.g., Sen and Manciu in Ref. [8]). We now show how it is possible to construct an infinite set of nonlinear force laws of the form $\mathcal{F} \propto z^{n}$, where $n \geqslant 1$, by tuning the contact geometry between two elastic objects while keeping a constant average pressure at fixed $z$. Dissipative and nondissipative nonlinear chains with arbitrary $n$ can be realized as very large scale integrated (VLSI) circuits [17], and preliminary studies are currently under way for the class of systems discussed here [7]. 
(a)

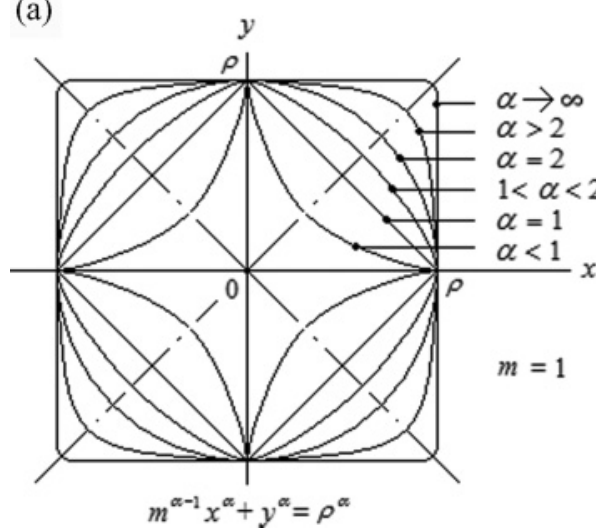

(b)

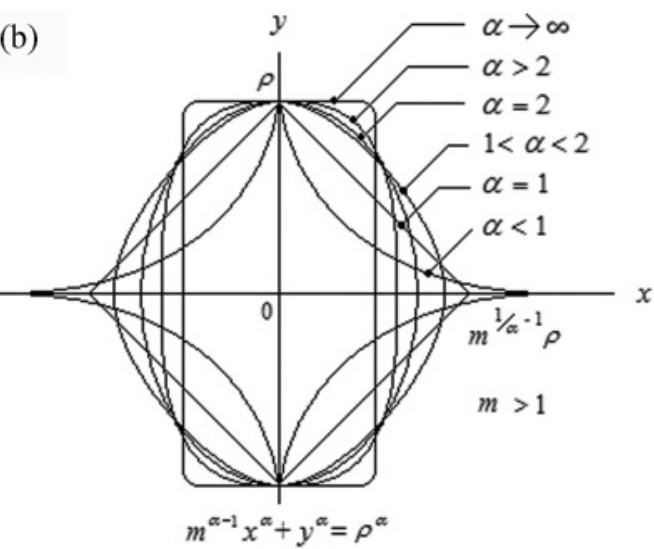

FIG. 1. (a) Cross-sectional areas along the $x-y$ plane for various values of $\alpha, m=1$. (b) Cross-sectional areas obtained for the same cases as in (a) but for $m>1$, where $m$ is eccentricity.

We start by noting that nonelliptic cross sections may be realized in contacts between solids with axial symmetry. We construct a treatment that relies on the cross-section geometry and elasticity theory. To this end, we introduce a generalized relation between $x, y$, and $z$ for various contact geometries pertaining to the two solids with axial symmetry of interest (see Fig. 1). We show that this generalized relation leads to a generalization of Hertz's law. Our result reproduces all known elastic force laws between two solids with axial symmetry up to a geometry-dependent constant factor and hence provides the conceptual framework for constructing systems with desired nonlinear force laws.

\section{CONTACT CROSS SECTION}

The centers of the bodies are placed along the $z$ axis. The radii of curvatures of these bodies near the contact surface are described as $R_{1}$ and $R_{2}$ and we invoke a factor $m$, for simplicity, to describe the aspect ratio in the two bodies. We claim now that

$$
\begin{aligned}
z_{1,2} & =\frac{x^{\alpha}}{R_{1,2}^{\alpha-1}}+\frac{y^{\alpha}}{\left(m R_{1,2}\right)^{\alpha-1}} \Rightarrow y^{\alpha}+m^{\alpha-1} x^{\alpha} \\
& =z_{1,2}\left(m R_{1,2}\right)^{\alpha-1} \equiv \rho^{\alpha},
\end{aligned}
$$

where $z_{1,2}$ is the grain compression for grain 1,2 and the overlap is defined as $z \equiv z_{1}+z_{2}$. In Eq. (1), $\alpha>0$ and $m>0$ and $m$ controls the aspect ratio of the cross-sectional areas as shown in Fig. 1. Observe that knowledge of $\rho$ implies knowledge of $a$ and $b$ in the Hertz theory that has been sketched above [4]. We assume $x, y \geqslant 0$ in Eq. (1) and will use the appropriate reflections for constructing the full cross section. This simplification reduces unnecessary algebra without any loss of generality. Observe that for $\alpha=2$ and $z=$ constant, Eq. (1) reduces to that of an ellipse. The quantity $\rho$ is a generalized radius of the cross-sectional area with its perimeter given by Eq. (1). Figure 2 illustrates the geometry along the axis of axial symmetry ( $z$ axis) with reference to $R_{1}, z_{1}$, and $\rho$.

\section{TRANSVERSE EXPANSION AND CROSS SECTION}

We expect that when two elastic bodies are in intimate contact and share a flat interface, they will both suffer some transverse expansion as shown by curve b in Fig. 2. This transverse expansion turns out to be important in constructing the derivation of the repulsive force law between two elastic bodies in contact in our approach. This correction can be introduced quite generally by assuming $\rho_{\text {new }}^{2}=\frac{m\left(R_{1}+R_{2}\right)}{Q} \rho$, where $\rho_{\text {new }}$ is the equivalent radius of the contact area with the transverse expansion. $Q$ enters naturally as a dimensionless parameter associated with the assertion that $\rho_{\text {new }}$ is proportional to $m,\left(R_{1}+R_{2}\right)$, and $\rho$. From an experimental standpoint, knowledge of $\kappa$ with and without transverse expansion would reveal $\rho$ and $\rho_{\text {new }}$ in our approach.

We can now introduce an equation which has the same form as Eq. (1) but uses scaled variables $X$ and $Y$ instead of $x$ and $y$ such that we retain the form of Eq. (1) while incorporating the transverse expansion as follows:

$$
Y^{\alpha}+m^{\alpha-1} X^{\alpha}=\left[\frac{m\left(R_{1}+R_{2}\right)}{Q} \rho\right]^{\alpha / 2} \equiv \rho_{\text {new }}^{\alpha} .
$$

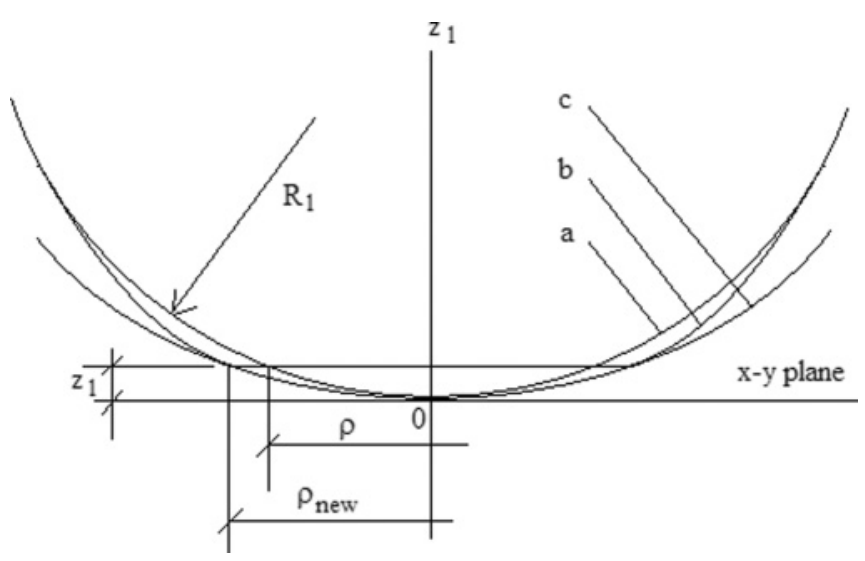

FIG. 2. Undistorted radius of curvature of one of the bodies is shown as curve a, the modification of the contact surface of the object deformed by $z_{1}$ is shown as curve $\mathrm{b}$, and curve $\mathrm{c}$ shows the curvature at the origin after the transverse expansion, indicated by $\rho_{\text {new }}$ [see Eq. (2)], has taken place. 
Once we have characterized the cross section, it is imperative that we understand how $\rho_{\text {new }}$ depends upon $z_{1}$ and $z_{2}$ (though Fig. 2 shows $z_{1}$ only, note that $z_{2}$ can be shown in a similar way). To this end we may write

$$
\begin{aligned}
z \equiv z_{1}+z_{2} & =\rho^{\alpha} m^{1-\alpha}\left(R_{1}^{1-\alpha}+R_{2}^{1-\alpha}\right) \\
& =\rho^{\alpha} m^{1-\alpha} \frac{R_{1}^{\alpha-1}+R_{2}^{\alpha-1}}{R_{1}^{\alpha-1} R_{2}^{\alpha-1}} .
\end{aligned}
$$

Making $\rho^{\alpha}$ the subject of our interest in Eq. (3) and using Eq. (2) we can write

$$
\rho_{\text {new }}^{2 \alpha}=\left[\frac{\left[m\left(R_{1}+R_{2}\right)\right]}{Q}\right]^{\alpha} m^{\alpha-1} \frac{\left(R_{1} R_{2}\right)^{\alpha-1}}{\left(R_{1}^{\alpha-1}+R_{2}^{\alpha-1}\right)} z .
$$

We are now in a position to study the cross-sectional area $A$ as a function of $z$, which turns out to be the crucial part of this calculation. Since the cross section is symmetric, we integrate over a quarter of the area,

$$
\begin{aligned}
A(z) & =4 \int_{X=0}^{X(Y=0)} Y(X) d X \\
& =4 \int_{X=0}^{\rho_{\text {new }} m^{\frac{1}{\alpha}-1}}\left[\rho_{\text {new }}^{\alpha}-m^{\alpha-1} X^{\alpha}\right]^{1 / \alpha} d X \\
& =4 m^{\frac{1}{\alpha}-1}\left(\rho_{\text {new }}^{2}\right)_{2} F_{1}\left[-\frac{1}{\alpha}, \frac{1}{\alpha} ; 1+\frac{1}{\alpha} ; 1\right],
\end{aligned}
$$

where ${ }_{2} F_{1}\left[-\frac{1}{\alpha}, \frac{1}{\alpha} ; 1+\frac{1}{\alpha} ; 1\right]$ is a hypergeometric function of $\alpha$. The above integral can be evaluated using the online Mathematica integrator or a similar utility. A series representation of this function is given as

$$
\begin{aligned}
{ }_{2} F_{1}[a, b ; c ; 1] & =1+\frac{a b}{1 ! c}+\frac{a(a+1) b(b+1)}{2 ! c(c+1)}+\cdots \\
& =\sum_{n}^{\infty} \frac{(a)_{n}(b)_{n}}{(c)_{n} n !}
\end{aligned}
$$

where the series converges if $c$ is a positive integer. In the above expression, $(\cdots)_{n}$ is Pochhammer's symbol and is written as

$$
(a)_{n} \equiv \frac{\Gamma(a+n)}{\Gamma(a)}=a(a+1) \cdots(a+n-1) .
$$

\section{GENERALIZED LAW}

To obtain the force associated with compressing the two elastic objects under consideration, we use standard elasticity theory [2] to write an expression for the change in averaged pressure,

$$
\frac{\Delta \mathcal{F}}{A(z)} \propto \frac{\Delta z}{h-z},
$$

where we consider $h$ to be the distance between the centers of the grains along the $z$ axis. At this point to express Eq. (8) we note that regardless of the nature of the stress on the elastic objects, the extensions along the directions of the axes and the



FIG. 3. Plot showing how the exponent $n$ in the force law depends on $\alpha$ in Eq. (1). For $n=2$ one obtains Hertz's law, whereas for $n \rightarrow \infty$ one obtains the force between two disks as discussed in the text.

normal tractions across planes at right angles are linked by equations of the following form (see Ref. [2], Chap. 3, p. 102):

$$
\epsilon_{z z}=\frac{1}{E}\left(\sigma_{z z}-v \sigma_{x x}-v \sigma_{y y}\right),
$$

etc., with $E$ as Young's modulus and $v$ as the Poisson ratio to describe the constrained compression along the $z$ axis. Also (see [3], p. 20)

$$
\sigma_{x x}=\sigma_{y y}=\frac{v}{2} \sigma_{z z} .
$$

Using Eq. (10) in Eq. (9) then leads to

$$
\sigma_{z z}=\frac{E}{1-v^{2}} \epsilon_{z z}
$$

which implies for $h>>z$ in Eq. (8) we can write

$$
\frac{\Delta \mathcal{F}}{A(z)}=\frac{E}{1-v^{2}} \frac{\Delta z}{h},
$$

and hence we find

$$
\mathcal{F}=\frac{E}{1-v^{2}} \int_{0}^{z} \frac{A\left(z^{\prime}\right)}{h} d z^{\prime},
$$

which implies

$$
\mathcal{F}=a z^{1+1 / \alpha}=a z^{n}
$$

where

$$
\begin{aligned}
a= & \frac{4}{Q} \frac{E}{\left(1-v^{2}\right)} \frac{m}{(1+1 / \alpha)} \frac{\left(R_{1}+R_{2}\right)}{h} \\
& \times\left[\frac{R_{1}^{\alpha-1} R_{2}^{\alpha-1}}{R_{1}^{\alpha-1}+R_{2}^{\alpha-1}}\right]^{1 / \alpha}{ }_{2} F_{1}\left[-\frac{1}{\alpha}, \frac{1}{\alpha} ; 1+\frac{1}{\alpha} ; 1\right] .
\end{aligned}
$$

Equation (15) describes the general form of the force law associated with elastic repulsion between two solids with axial symmetry. The contact surface is described by parameters $\alpha$ and $m$ in Eq. (1) and the exponent of the nonlinear law depends only on $\alpha$. We show below that for $\alpha=2$ and spherical $(m=1)$ grains, we recover Hertz's law; while for the case of contact 




FIG. 4. Schematic of how grains satisfying two different force laws on opposite ends may be constructed. Note that in this case $h=R_{1}+R_{2}+a$.

between two disks, we recover the linear force law. Figure 3 relates the force law $\mathcal{F}=a z^{1+1 / \alpha}$ with the shapes of those identical elastic solids that would relate to the contact interface for the specific $\alpha$ value.

\section{FORCE LAW BETWEEN SPHERES}

For spherical grains, we set $\alpha=2$ and $m=1$ in Eqs. (1) and (2), and with reference to Fig. 4 define $h \equiv R_{1}+R_{2}$, which yields

$$
\begin{aligned}
F & =\frac{1}{Q} \frac{2}{3} \frac{4 E}{\left(1-v^{2}\right)}\left[\frac{R_{1} R_{2}}{R_{1}+R_{2}}\right]^{1 / 2}{ }_{2} F_{1}\left[-\frac{1}{2}, \frac{1}{2} ; \frac{3}{2} ; 1\right] z^{3 / 2} \\
& =\frac{\pi}{Q} \frac{2}{3} \frac{E}{\left(1-v^{2}\right)}\left[\frac{R_{1} R_{2}}{R_{1}+R_{2}}\right]^{1 / 2} z^{3 / 2} .
\end{aligned}
$$

It may be puzzling to see the quantity $Q$ in the prefactor on the right-hand side of Eq. (16). A closer look reveals that a very similar quantity, $\kappa=a / b$, also enters the derivation of the prefactor in the typical derivation of Hertz's law as presented in detail in Ref. [4] and sketched earlier in this paper. In the typical derivation, $\kappa$ needs to be determined from the actual system geometry at hand. In the present treatment, $Q$ needs to be determined by observing the bulging effect of the grains near the contact region as shown schematically by curve b in Fig. 2 [see the discussion below Eq. (16) in Leroy's paper in Ref. [5] in this connection). Here, unlike in the existing treatments of the Hertz problem, we have assumed that the force across the entire contact region is only $z$ dependent rather than assuming that the pressure in the contact surface is $x, y$ dependent as introduced by Hertz [1]. It turns out that an assumption regarding the $x, y$ dependence of pressure at the contact surface is not necessary if one uses Eqs. (1) and (2) to derive Hertz's law. What is, however, important is that Eq. (1) provides the option to introduce nonelliptical contact interfaces using two parameters $\alpha$ and $m$. The transverse expansion at the interface of two soft elastic solids can be accounted for by defining $\rho_{\text {new }}$. The quantity $\rho_{\text {new }}$ can be used to successfully calculate the correct contact area described in Eq. (2). Thus, it is critical that when the area integration is performed, expected results are recovered. We address this point below.

The factor $\pi$ in Eq. (16) should be understood as follows. Indeed, the cross-sectional area for $\alpha=2, m=1$ (i.e., circular contact) in Eq. (5) yields $A=4 \rho_{\text {new } 2}^{2} F_{1}\left[-\frac{1}{2}, \frac{1}{2} ; \frac{3}{2} ; 1\right]=4 \times$ $\frac{\pi}{4} \rho_{\text {new }}^{2}$ as expected. Hence $\pi$ enters because of the geometry of the contact surface.

\section{FORCE LAW BETWEEN DISKS}

Let us now turn our attention to the case of two disks of radius $R$, which would lead to a rectangular cross section in contact. This case is realized when $\alpha \rightarrow \infty$ in Eq. (3) above (see Fig. 1). Setting $R_{1}=R_{2}=R, h=2 R$, and $\alpha \rightarrow \infty$, and using ${ }_{2} F_{1}[0,0 ; 1 ; 1]=1$, Eq. (15) becomes

$$
a=\frac{2 m}{Q} \frac{E R}{1-v^{2}},
$$

and

$$
\mathcal{F}=a z,
$$

which is the expected linear force law $\mathcal{F}=a z$ that characterizes the force between two disks as expected. We did not require the radius to diverge in our formulation. Rather, the disk contact was achieved via $\alpha \rightarrow \infty$ as shown in Fig. 1.

Given that the studies presented here concern two elastic half-solids, one can imagine constructing "designer grains" (see Fig. 4) where adjacent granular contacts may be made to vary if needed.

\section{CONCLUSION}

To summarize, we have developed a connection between the geometry of the contact region between two elastic objects with axial symmetry [given by Eq. (1)] and the magnitude and behavior of the repulsive force between these two objects. The contact region is perpendicular to the axis joining the centers of the objects [Eq. (15)]. We recover Hertz's law for two spheres in contact and the linear force law for two disks in contact. The ability to control the forces between adjacent elastic objects is expected to introduce novel possibilities in energy transport, dispersion, and localization in granular systems $[7,8,16]$. Further, the solutions of dynamical equations of motion for designer grains in granular alignments can be realized using VLSI circuits [17]. Hence, this work raises the possibility of exploring systems with novel nonlinear potentials and across parameter ranges that may not necessarily have known physical significance.

\section{ACKNOWLEDGMENTS}

S.S. thanks Prof. Robert W. Newcomb and a referee for valuable comments. D.S. and S.S. were supported by the US Army Research Office. C.D. was supported by the US Army Research Office and an NSF CAREER grant. 
[1] H. Hertz, J. Reine Angew. Math. 92, 155 (1881).

[2] A. E. H. Love, A Treatise on the Mathematical Theory of Elasticity (Dover, New York, 1944).

[3] L. D. Landau and E. M. Lifshitz, Theory of Elasticity (Pergamon, Oxford, 1970).

[4] W. Goldsmith, Impact (Edward Arnold, London, 1960).

[5] I. N. Sneddon, Int. J. Eng. Sci. 3, 47 (1965); D. A. Spence, Proc. R. Soc. London A 305, 55 (1968); F. Hermann and M. Seitz, Am. J. Phys. 50, 977 (1982); B. Leroy, ibid. 52, 230 (1984).

[6] K. L. Johnson, Contact Mechanics (Cambridge University, Cambridge, England, 1985).

[7] V. Nesterenko, J. Appl. Mech. Tech. Phys. 5, 733 (1983); A. N. Lazaridi and V. Nesterenko, ibid. 26, 405 (1985); V. Nesterenko, Dynamics of Heterogeneous Materials (Springer, New York, 2001), and references therein.

[8] R. S. Sinkovits and S. Sen, Phys. Rev. Lett. 74, 2686 (1995); S. Sen and R. S. Sinkovits, Phys. Rev. E 54, 6857 (1996); C. Coste, E. Falcon, and S. Fauve, ibid. 56, 6104 (1997); S. Sen, M. Manciu, and J. D. Wright, ibid. 57, 2386 (1998); S. Sen and M. Manciu, ibid. 64, 056605 (2001); For a recent review, see S. Sen et al., Phys. Rep. 462, 21 (2008).

[9] C. H. Liu and S. R. Nagel, Phys. Rev. Lett. 68, 2301 (1992); H. Jaeger et al., Rev. Mod. Phys. 68, 1259 (1996); X. Jia,
Phys. Rev. Lett. 93, 154303 (2004); Th. Brunet, ibid. 101, 138001 (2008); L. Bonneau, Phys. Rev. E 75, 016602 (2007).

[10] J. MacDonald and J. R. Lockwood, Alternatives for Landmine Detection (RAND, Santa Monica, 2003); S. Sen et al., Int. J. Mod. Phys. B 19, 2951 (2005).

[11] A. Sokolow, J. M. M. Pfannes, R. Doney, M. Nakagawa, J. H. Agui, and S. Sen, Appl. Phys. Lett. 87, 254104 (2005).

[12] See, for instance, Y. Hiraiwa and Y. Kasubuchi, Eur. J. Soil Sci. 51, 211 (2000), and references therein.

[13] G. BenDor et al., Exp. Fluids 22, 432 (1997); G. Baker et al., Bull. Seismol. Soc. Am. 90, 494 (2000); Th. Brunet et al., Geophys. Res. Lett. 35, L19308 (2008); L. Gilcrist et al., Appl. Phys. Lett. 91, 254103 (2007).

[14] G. Fu, J. Appl. Mech. 74, 373 (2007); M. Ciamarra, A. H. Lara, A. T. Lee, D. I. Goldman, I. Vishik, and H. L. Swinney, Phys. Rev. Lett. 92, 194301 (2004).

[15] G. G. Adams and M. Nosonovsky, Tribol. Int. 33, 431 (2000).

[16] E. Fermi, J. Pasta, and S. Ulam, Los Alamos National Laboratory, Rep. No. LA-1940, 1955 (unpublished); J. Ford, Phys. Rep. 213, 272 (1984); D. K. Campbell, S. Flach, and Y. Kivshar, Phys. Today 57, 43 (2004).

[17] See, for example, R. W. Newcomb and J. D. Lohn, in Handbook of Brain Theory and Neural Networks, edited by M. Arbib (Bradford Books, MIT Press, Cambridge, MA, 1995). 